\title{
Comparative Study of Antioxidant Activity of Apple and Pear Peel
}

\author{
Kyung Soon Kim and Kwang Soo Roh* \\ Department of Biology, Keimyung University, Daegu 704-701, Korea
}

\begin{abstract}
Apple and pear are popular fruits consumed in Korea and are common fruit in daily diet. In order to compare the antioxidant activity of the apple and pear peels, total polyphenol contents, total flavonoid contents, $\mathrm{ABTS}^{+}$free radical scavenging activity, and DPPH free radical scavenging activity were measured from hot water, ethanol, and methanol extracts of the two fruit peels. The total polyphenol and flavonoid contents were highest in $95 \%$ methanol extracts of the apple peelsand $70 \%$ ethanol extract of the pear peels, respectively. Total polyphenol contents of the pear peels were higher than that of apple peels, and total flavonoid contents of the apple peels were higher than that of pear peels. The apple and pear peels had the highest $\mathrm{ABTS}^{+}$and DPPH free radical scavenging activity in $95 \%$ methanol extracts and $70 \%$ ethanol extracts, respectively. ABTS ${ }^{+}$and DPPH free radical scavenging activity of pear peels was higher than that of apple peels, and the DPPH free radical scavenging activity of apple and pear peels were detected in hot water, $95 \%$ methanol, and $70 \%$ ethanol extracts, respectively. Ascorbic acid, a synthetic antioxidant used as positive control, had significantly higher scavenging activity than the apple and pear peels. In conclusion, the apple and pear peelshave great potential as natural antioxidants. Therefore, above results should be considered to provide the possibility for the development of high functional antioxidants.
\end{abstract}

Key words - ABTS, Apple peel, DPPH, Flavonoid, Pear peel, Polyphenol

\section{Introduction}

Apple, one of the most popular and important fruits in the world, occupies about 45 percent of the total area under fruit crops and the largest amount of production among the fruits in Korea (Lee, 2000). Itis also rich in nutritional ingredients such as vitamin $\mathrm{C}$, dietary fibers, carbohydrates as well as functional ingredients like phenol ingredients, effective against diseases such as diabetes, cancers, cerebrovascular diseases, and heart vascular diseases (Kroon and Williamson, 2005). It is not only popular as a fruit itself, but also used as processed food such as drinks, jam, canned and dried foods (Boyer and Liu, 2004).

Korean pear that belongs to Pyrus genus of the rose family is a deciduous arborescent treeand mostly grown in the middle and southern parts of Korean peninsular (Hong et al., 2004). It is an important fruit next to apple, citrus fruit and grapes (Zhang et al., 2003). It has been reported that the cell membranes of pear contain large amount of moisture and is

*Corresponding author. E-mail : rks@kmu.ac.kr composed of $20-30 \%$ of cellulose, $25 \%$ of hemi- cellulose, $35 \%$ of pectin, $5-10 \%$ of glycoprotein and small amount of phenolic substances which are mutually connected in complicated structures (Fisher and Bennett, 1991). In ancient folk remedies, the peels are used as healing herbs for boil and skin diseases, flesh for treating constipation, cough, phlegm and hangover, and pear leaves as treatment for the acute gastroenteritis as a special remedy (Jang et al., 2003).

Polyphenols that are contained much in apple and pear are antioxidation ingredients, and it has been reported that phenol contents in the peel is about 2-9 times more than in the flesh (Tsao et al., 2003). Polyphenol is an aromatic compound which has more than two phenolic hydroxyl groups in a molecule, and it has been reported that polyphenol is concerned in strengthening immunity (Choi et al., 2003) and bacterial activity (Boo et al., 2012). The natural polyphenol compounds, which are plenty in plants, are majorly flavonoids, lignins, and tannins (Sharma and Sehgal, 1992).

Reactive oxygen species (ROS) produced during the metabolic processing of various bioenergies are noxious materials which are also produced in all organisms including human 
beings by free radical reaction, and degenerate biofunctions such as DNA degeneration, damaging cell membranes, and protein oxidation. ROS causes various adult diseases and accelerates aging (Valko et al., 2007). Antioxidants such as ascorbic acid, tocopherol and carotenoid, play important roles in delaying or inhibiting the degeneration of biofunction (Lim et al., 1996). The development of natural antioxidants has been undertaking continuously (Cort, 1974), but it requires high costs. The antioxidation effects of the natural antioxidants are lower than those of synthetic antioxidants. On the other hand, since the antioxidation effects of synthetic antioxidants such as butyloted hydroxyanisol (BHA) and butylated hydroxytoluene (BHT) are known to be higher and prices are also low, they are used largely in commercial purposes. However, these substances are harmful to human body, and hence studies on the natural antioxidants with high safety and antioxidation activity are continuously requested (Branen, 1975).

Therefore, the objectives of this study were (1) to determine whether apple and pear peels can be used as antioxidants, and (2) to compare the antioxidation effects by measuring the total polyphenol contents, total flavonoid contents, ABTS free radical scavenging activity and DPPH free radical scavenging activity for different solvents hot water, $70 \%$ ethanol and 95\% methanol.

\section{Materials and Methods}

\section{Materials}

The apple (trade name: Hongro) and pear (trade name: Singo) grown in Geochang Gyeongnam and Sangju Gyeongbuk respectively, were purchased from the local market, and only peels were used asmaterials in our study. 1,1-Diphenyl2-picrylhydrazyl (DPPH) was purchased from Fluka Biochemika AG (Buchs, Switzerland). 2,2'-Azino-bis (3-ethylbenzthiazoline-6-sulfonic) acid (ABTS), ascorbic acid,and other chemicals were purchased from Sigma (St Louis, MO, USA). All chemicals were of analytical grade.

\section{Preparation of the extracts}

The apple and pear peels were dried in a vacuum oven at $40^{\circ} \mathrm{C}$, ground into fine powder, and filtered using the sieve. For hot water extraction, $10 \mathrm{~g}$ of each powder sample was boiled in $500 \mathrm{~mL}$ of distilled water at $100^{\circ} \mathrm{C}$ for $2 \mathrm{hrs}$ using a heating mantle (Wisd, Korea), and then cooled and centrifuged at $8,000 \mathrm{rpm}$ for $15 \mathrm{mins}$. The pellet collected by centrifugation was resuspended in $500 \mathrm{~mL}$ of distilled water, and centrifuged at 8,000 rpm for $15 \mathrm{mins}$. For ethanol and methanol extractions, after $10 \mathrm{~g}$ of each powder samples were soaked in $500 \mathrm{~mL}$ of $70 \%$ ethanol and $95 \%$ methanol, respectively, extracted at $25^{\circ} \mathrm{C}$ for $6 \mathrm{hrs}$ using shaking incubator (Hanbaek, Korea) at $120 \mathrm{rpm}$, and then centrifuged at 8,000 rpm for 15 mins.

The supernatant solutions collected by three times centrifugation at $8,000 \mathrm{rpm}$ for $15 \mathrm{mins}$, concentrated until $10 \mathrm{ml}$ remained using a rotary evaporator (Eyela, Japan). The concentration temperature was at $65^{\circ} \mathrm{C}$ for hot water extraction and $50^{\circ} \mathrm{C}$ for ethanol and methanol extractions. Finally, freeze dried samples were stored at $-70^{\circ} \mathrm{C}$ until further analysis.

\section{Determination of total extraction yields}

Total extraction yields of the apple and pear peels were calculated by the following equation:

$$
\text { Yield }(\%)=\mathrm{A} / \mathrm{B} \times 100
$$

A is amount of samples after freeze dry, and B is amount of samples used for extraction.

\section{Determination of total polyphenol contents}

The total polyphenol contents of the peel extracts of apple and pear were determined by Folin-Denis method (Folin and Denis, 1912). $60 \mu \mathrm{L}$ of Folin- Ciocalteu reagent were added to $60 \mu \mathrm{L}$ of the different extract solutions, and then stand for three min. Thereafter, $60 \mu \mathrm{L}$ of $10 \% \mathrm{Na}_{2} \mathrm{CO}_{3}$ was added to the mixture and then $1 \mathrm{hr}$ incubation at room temperature, the absorbance was determined at $750 \mathrm{~nm}$ using ELISA microplate reader (Bio-Rad 680, USA).Gallic acid monohydrate as a standard phenol was used to prepare a standard curve. The total polyphenol contents of the extracts were expressed as gallic acid equivalents from the linear regression curve of gallic acid

\section{Determination of total flavonoid contents}

The total flavonoid contents of apple and pear peel extracts 
were determined by the method of Moreno et al. (2000). 62.5 $\mu \mathrm{L}$ of the different extract solutions were added to each well of plate, followed by addition of $1.08 \mathrm{~mL}$ of $80 \%$ ethanol, 30 $\mu \mathrm{L}$ of $10 \%$ aluminum nitrate, and $30 \mu \mathrm{L}$ of $1 \mathrm{M}$ potassium acetate, mixed well and reacted at room temperature for 40 mins. Absorbance of the reaction mixture was read at $415 \mathrm{~nm}$ using microplate reader. Rutin hydrate as a standard flavonoid was used to prepare the standard curve. Total flavonoid contents were expressed as Rutin Equivalents from the linear regression curve of rutin.

\section{ABTS radical scavenging assay}

Antioxidant activity of the peel extracts of apple and pear were measured in this assay as ability to ABTS radical scavenge according to Re et al. (1999). $\mathrm{ABTS}^{+}$was produced by reacting $7 \mathrm{mM} / \mathrm{L}$ of ABTS [2,2'-azino-bis(3-etylbenzothiazoline- $\delta$-sulfonic acid)] solution with $2.45 \mathrm{mM} / \mathrm{L}$ of potassium persulfate, and the mixture would be kept in the dark at room temperature for $24 \mathrm{hrs}$. $\mathrm{ABTS}^{+}$solution was diluted with PBS (phosphate buffer saline) to an absorbance of $0.70 \pm 0.02$ at $732 \mathrm{~nm} .20 \mu \mathrm{L}$ of the different extract samples diluted to $1000 \mu \mathrm{g} / \mathrm{mL}$ were added to $180 \mu \mathrm{L}$ of $\mathrm{ABTS}^{+}$ solution and mixed vigorously. After reaction at room temperature for $1 \mathrm{~min}$, the absorbance at $732 \mathrm{~nm}$ was measured using microplate reader. Ascorbic acid as a synthetic antioxidant, prepared in the same concentrations as the test extracts, was used as the positive controls for comparison. The ABTS ${ }^{+}$scavenging effect was calculated by the following formula:

$$
\begin{aligned}
& \text { Electron donating activity }(\%) \\
& =(1 \text {-Asample/Acontrol }) \times 100
\end{aligned}
$$

$\mathrm{A}_{\text {control }}$ is the absorbance of control without sample and $\mathrm{A}_{\text {sample }}$ is the test sample with $\mathrm{ABTS}^{+}$.

\section{DPPH radical scavenging assay}

Antioxidant activity of the peel extracts of apple and pear were measured in this assay as ability to DPPH radical scavenge according to Blois (1958). $160 \mu \mathrm{L}$ of the different extract samples diluted to $1,000 \mu \mathrm{g} / \mathrm{mL}$ were added to $40 \mu \mathrm{L}$ of 0.15 mM DPPH (1,1-diphenyl-2-picrylhydrazyl) solution, respectively and allowed to react in the dark at room temperature for $30 \mathrm{~min}$. Absorbance of DPPH was measured at 517 nmusing microplate reader. Ascorbic acid prepared in the same concentrations as the test extracts was used as the positive controls for comparison. DPPH free radical scavenging activity was calculated according to the following equation:

$$
\begin{aligned}
& \text { Electron donating activity }(\%) \\
& =(1-\text { Asample/Acontrol }) \times 100
\end{aligned}
$$

$\mathrm{A}_{\text {control }}$ is the absorbance of control without sample and $\mathrm{A}_{\text {sample }}$ is the test sample with DPPH

\section{Statistical analysis}

Activity data were analyzed using a one-way analysis of variance (ANOVA) accompanied with Tukey tests and Student's $t$-test (SPSS for Windows, Ver. 21), and presented as means \pm standard error. All measurements were replicated three times. P-values less than 0.05 were considered to be statistically significant.

\section{Results}

\section{Total extraction yields of apple and pear peels}

In apple peels, total extraction yields from the hot water, $70 \%$ ethanol, and $95 \%$ methanol were $59.91 \%, 81.94 \%$, and $51.25 \%$, respectively (Table 1). In pear peels, total extraction yields were $46.84 \%, 59.22 \%$, and $46.09 \%$, respectively

Table 1. Total extraction yields of apple peels

\begin{tabular}{cc}
\hline Sample & Total extraction yields (\%) \\
\hline Water extracts & 59.91 \\
$70 \%$ EtOH extracts & 81.94 \\
$95 \%$ MtOH extracts & 51.25 \\
\hline
\end{tabular}

Table 2. Total extraction yields of pear peels

\begin{tabular}{cc}
\hline \hline Sample & Total extraction yields (\%) \\
\hline Water extracts & 46.84 \\
$70 \%$ EtOH extracts & 59.22 \\
$95 \%$ MtOH extracts & 46.09 \\
\hline
\end{tabular}


(Table 2). In both apple and pear peels, the highest extract yields were observed in $70 \%$ ethanol extract, and higher in the apple peel extracts.

\section{Total polyphenol contents}

The total polyphenol contents in apple peels were $16.41 \pm$ $0.23 \mathrm{mg} / \mathrm{g}, 15.32 \pm 0.09 \mathrm{mg} / \mathrm{g}$, and $16.50 \pm 0.12 \mathrm{mg} / \mathrm{g}$ from the hot water, $70 \%$ ethanol and 95\% methanol extracts, respectively. Statistically significant lower yields were observed in $70 \%$ ethanol extracts as compared to hot water and $95 \%$ methanol extracts, while the latter two showed no difference. In the case of pear peels, the contents from the hot water, $70 \%$ ethanol and $95 \%$ methanol extracts were $21.68 \pm 0.03 \mathrm{mg} / \mathrm{g}$, $24.08 \pm 0.25 \mathrm{mg} / \mathrm{g}$, and $22.17 \pm 0.08 \mathrm{mg} / \mathrm{g}$, respectively. Statistical significance was similar to the apple peel samples (Fig. 1). The total polyphenols were maximized in $95 \%$ methanol extracts ofthe apple peels, and $70 \%$ ethanol extracts of the pear peels. The total polyphenol contents were higher in the pear peels than in the apple peels.

\section{Total flavonoid contents}

The total flavonoid contents in apple peels from hot water, $70 \%$ ethanol, and $95 \%$ methanol were $15.08 \pm 0.21 \mathrm{mg} / \mathrm{g}$, $16.15 \pm 0.43 \mathrm{mg} / \mathrm{g}$, and $22.52 \pm 1.16 \mathrm{mg} / \mathrm{g}$, respectively. The highest content was observed in $95 \%$ methanol extracts. In the case of pear peels, the observed contents were $15.18 \pm 0.49$ $\mathrm{mg} / \mathrm{g}, 17.04 \pm 1.32 \mathrm{mg} / \mathrm{g}$, and $14.03 \pm 0.31 \mathrm{mg} / \mathrm{g}$, respectively. The highest content was observed in $70 \%$ ethanol extracts (Fig. 2). In apple peels, a significantly higher flavonoid contents were observed in 95\% methanol extracts as compared to those from hot water and $70 \%$ ethanol extracts. No such differences were observed in the pear peels.

\section{ABTS free radical scavenging activity}

ABTS free radical scavenging activity of ascorbic acid was $94.29 \pm 0.03 \%$, which indicates very high free radical scavenging activity. In the apple peel, ABTS free radical scavenging activity of the hot water, $70 \%$ ethanol and $95 \%$ methanol extracts were $19.14 \pm 9.57 \%, 10.56 \pm 5.28 \%$, and $20.29 \pm 10.14 \%$, respectively. The highest ABTS free radical scavenging activity was observed in $95 \%$ methanol extracts. ABTS free radical scavenging activity in the pear peel extracts were $39.83 \pm 19.91 \%, 44.16 \pm 22.08 \%$, and $43.50 \pm$ $21.75 \%$, respectively. The pear peels had the highest ABTS ${ }^{+}$ free radical scavenging activity in $70 \%$ ethanol extracts. ABTS free radical scavenging activity of pear peels was higher than that of apple peels (Fig. 3). Statistical significant results were not observed among the extracts in both apple and pear peels, which implies low scavenging activity in comparison with ascorbic acid.

\section{DPPH free radical scavenging activity}

DPPH free radical scavenging activity of ascorbic acid was $34.23 \pm 1.59$, and DPPH free radical scavenging activity of apple peels from hot water, $70 \%$ ethanol and $95 \%$ methanol were, respectively, $32.90 \pm 1.15 \%, 23.78 \pm 1.55 \%$, and $38.93 \pm$ $1.70 \%$, with $95 \%$ methanol being the highest scavenging activity. DPPH free radical scavenging activity of pear peels were $38.51 \pm 10.64 \%, 67.92 \pm 13.86 \%$, and $49.83 \pm 4.66 \%$ for the same order of extracts. The $70 \%$ ethanol solvent showed

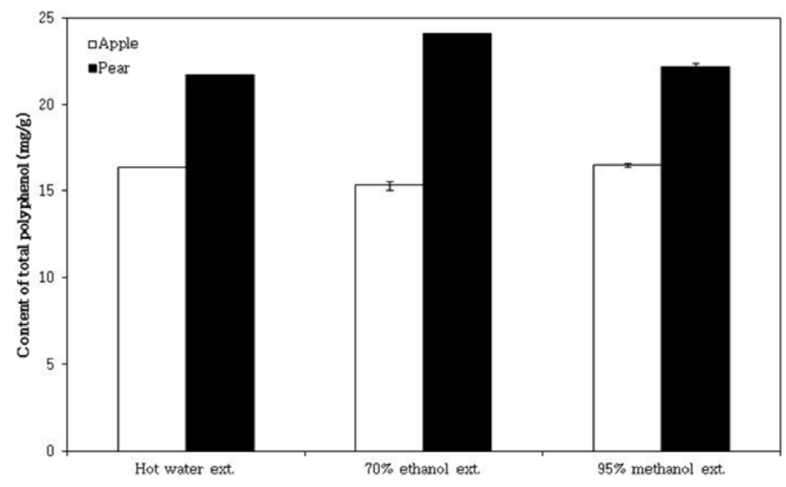

Fig. 1. Total polyphenol contents of apple and pear peels by solvents. The bars mean the standard error.

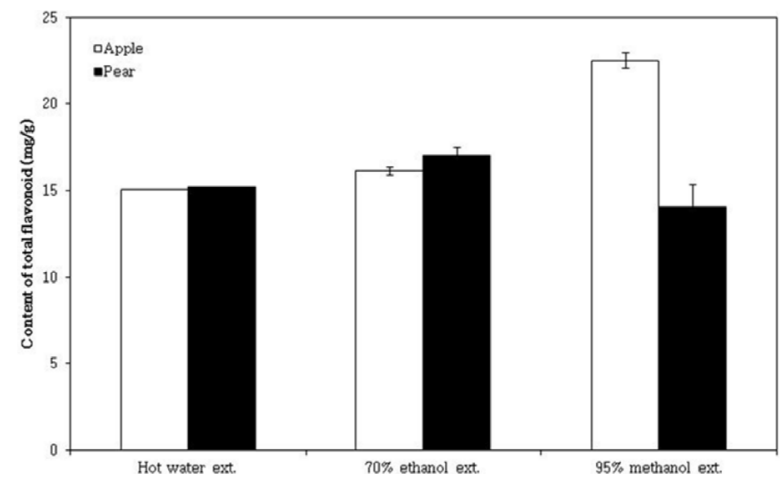

Fig. 2. Total flavonoid contents of apple and pear peels by solvents. The bars mean the standard error. 


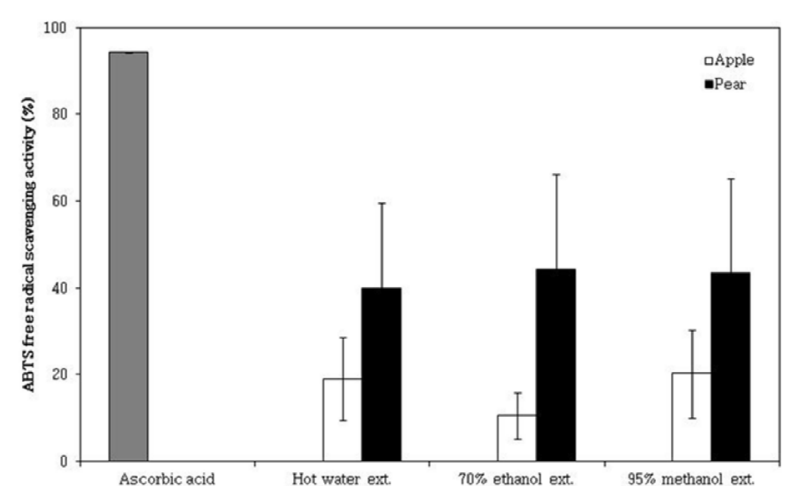

Fig. 3. ABTS radical scavenging activity of apple and pear peels by solvents. Ascorbic acid prepared in the same concentrations as the test extracts was used as the positive controls for comparison. The bars mean the standard error.

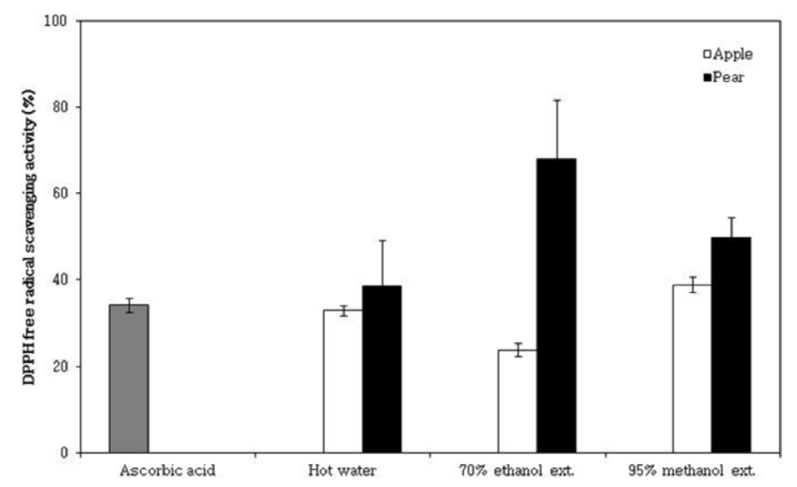

Fig. 4. DPPH radical scavenging activity of apple and pear peels by solvents. Ascorbic acid prepared in the same concentrations as the test extracts was used as the positive controls for comparison. The bars mean the standard error.

the highest scavenging activity (Fig. 4). In apple peels, the hot water showed statistically significant differences in DPPH free radical scavenging activity from $70 \%$ ethanol and $95 \%$ methanol, and in pear peels significant differences were observed between the hot water and $70 \%$ ethanol, and between the hot water and 95\% methanol. Comparisonwith ascorbic acid, statisticalsignificant results for DPPH free radical scavenging activity were not observed among the extracts in apple peels, whereas observed in $70 \%$ ethanol and $95 \%$ methanol extracts of pear peels.

\section{Discussion}

Our society is being progressed rapidly into aging society as the levels of living and medical care are elevated. Accordingly, peoples are more and more interested in preventing infectious diseases and/or delaying the aging. Therefore, the necessity of antioxidation is being increased. Thus, present study aims at finding out the valuable resources for antioxidation and possibilities of practical uses of these resources by studying the effects of antioxidants in the peels of our representative fruits, apple and pear (Zhang et al., 2003), widely distributed all over our country. Total extractionyields from $70 \%$ ethanol were highest in both apple and pear peels, and the yields were in the order of $70 \%$ ethanol, hot water and $95 \%$ methanol. Total extractionyields were higher in apple peels than in pear peels. However, it cannot be concluded that higher yield means higher antioxidation effect.

Phenolic compounds in plants, one of secondary metabolites, are known to have antioxidation function which is the property that phenolic hydroxyl group combines with large molecules such as enzymes (Husain et al., 1987), and hence experiments on the total polyphenol contents, based on the oxidoreduction reactions, are performed, applying the phenomenon of changing the color blue when the extracts are reduced by polyphenol compounds (Ainsworth and Gillespie, 2007). Wolfe et al. (2003) reported that antioxidation effect in the peel of apple was higher than in the flesh. Thereafter, inthe present study the total polyphenol contents were measured from apple peels only. The total polyphenol contents for the solvents did not show statistically significant differences although relatively higher polyphenol content was observed from $95 \%$ methanol extract. However, in apple peels the polyphenol contents from methanol extract was lower than $19.7 \pm 1.3 \%$ reported by Cheign et al. (2011). In pear peels, relatively higher polyphenol content was observed in 70\% ethanol extract. Park et al. (2007) reported that the polyphenol contents were determined from ethanol extracts of some Korean pear varieties. In the present study, relatively higher values of polyphenol were observed. As shown in a study on antioxidant activity of different parts of kiwi (Park et al., 2008), flavonoid is yellowish and contained more in the peels rather than in the flesh. In apple peels, relatively high values of flavonoid, which can function as an antioxidant, were observed in $95 \%$ methanol extracts, and in pear peels in $70 \%$ ethanol extracts. It can be seen that the total 
contents of polyphenol and flavonoid have the same trends. Similar results were reported in the pericarps of citron and trifoliate orange (Park et al., 2008). Similar results were also obtained in the present study.

ABTS free radical scavenging activity - a means of measuring antioxidation effects by using decolorizing reaction of bluish green color when ABTS cation absorbance produced by reacting with an oxidant, potassium persulfate, is scavenged (Myung and Hwang, 2008) - was used, and for which ascorbic acid were involved in the experiments. The higher scavenging activity was observed in ascorbic acid. In apple peels, the highest ABTS free radical scavenging activity was observed in $95 \%$ methanol extracts, and in pear peels it was highest in $70 \%$ ethanol extracts. According to the results of a study on antioxidant extracted from litchi peel (Jeong et al., 2010), the radical scavenging activity was increased as the concentrations of extracts increased. Thus, it is considered that, in order to affirm the radical scavenging activity, precise examinations should be made at several levels by dividing the part that gives the highest scavenging activity such that distance between levels is small.

DPPH free radical scavenging activity - a means of measuring antioxidation effects by using the phenomenon: decolorizing reaction of purple color and scavenging free radicals when a certain extract containing antioxidant activity donates an electron to DPPH free radical - was used (Yen and Chen, 1995), and ascorbic acid, a synthetic antioxidant, was used as positive control in the measurement of DPPH free radical scavenging activity. DPPH free radical scavenging activity was lower than ABTS free radical scavenging activity in ascorbic acid. Relatively high scavenging activities were observed from $95 \%$ methanol extract in apple peels and from $70 \%$ ethanol extract in pear peels. Scavenging activity was higher in pear peels than in apple peels. Considerably higher scavenging activities were observed from $70 \%$ ethanol extract and 95\% methanol extracts as compared to those from ascorbic acid, a synthetic antioxidant. Efficient substance of pomegranate for antioxidation was observed in the peels (Roh et al., 2005), and in a study on the antioxidant activity of Codonopsis lanceolate, the highest antioxidant activity was observed in the peels (Kang, 2009). However, according to the reports of Park et al. (2007) on the measurement of the antioxidant activity observed in ethanol extracts from Korean pears varieties, the antioxidant activityfrom whole fruits and flesh + peel were higher than those measured from the peel, which are different from the results reported by Jin and Song (2012), where the antioxidant activity in the peel was higher than in the flesh. The other study showed that free radical scavenging activity increased as the concentration of the extract from litchi peels increased (Jeong et al., 2010), and free radical scavenging activity of the extracts from Campbell grape seeds and peels increased along with the extract temperature (Park et al., 2003). Further studies are demanded to ascertain the freeradical scavenging activities and to find out the optimum levels or combination of levels of extracting temperature and of solvent concentrations that gives the highest activity through well-designed experiments.

Consequently, the total polyphenol contents and the total flavonoid contents were similar in values, showing that, on average, higher polyphenol was observed in pear peels and higher flavonoid in apple peels. In the measurement of electron donating activity, ABTS and DPPH free radical scavenging activity were, on average, higher in pear peels than in apple peels. Since the compatible solvents are different for different fruits, it is considered that the solvents may affect the antioxidant activities. Pear peels are considered more valuable than apple peels for the development of natural antioxidants.

\section{Acknowledgements}

This work is supported by Research Scholarship of Graduate School of Keimyung University in 2012.

\section{Literature Cited}

Ainsworth, E.A. and K.M. Gillespie. 2007. Estimation of total phenolic content and other oxidation substrates in plant tissues using Folin-Ciocalteu reagent. Natl. Proto. 2:875-877.

Blois, M.S. 1958. Antioxidant determinations by the use of a stable free radical. Nature 181:1198-1200.

Boo, H.O., J.S. Shin, S.J. Hwang, C.S. Bae and S.H. Park. 2012. Antimicrobial effects and antioxidative activities of the cosmetic composition having natural plant pigments. Korean 
J. Plant Res. 25:80-88 (in Korean).

Boyer, J. and R.H. Liu. 2004. Apple phytochemicals and their health benefits. Nutr. J. 3:1-15.

Branen, A.L. 1975. Toxicology and biochemistry of butylated hydroxyanisole and butylated hydroxytoluene. J. American Oil Chem. Soc. 52:59-63.

Cheigh, C.I., S.Y. Yoo and M.S. Chung. 2011. Efficient flavonoid extraction from apple peel by subcritical water and estimation of antioxidant activity. J. Med. Food 24:458-463.

Choi, H.J., H.S. Han, J.H. Park, J.H. Park, J.H. Bae, H.S. Woo, B.J. An, M.J. Bae, H.G. Kim and C. Choi. 2003. Effect of polyphenol compounds from Korean pear on immunofuntional activity. Korean J. Food Cult. 18:303-310.

Cort, W.M. 1974. Antioxidant activity of tocopherol and ascorbylpalmitate and their mode of action. J. American Oil Chem. Soc. 51:321-325.

Fisher, R.B. and A.B. Bennett. 1991. Role of cell wall hydrolase in fruit ripening. Ann. Rev. Plant Mol.Biol. 42:675-703.

Folin, O. and W. Denis. 1912. On phosphotungstic-phosphomolybdic compounds as color reagents. J. Biol. Chem. 12:239243

Hong, S.S., Y.P. Hong, B.S. Im, D.S. Jeong and I.S. Shin. 2004. Influence of picking stage storage type on the fruit respiration change and panel test in 'Wonwang', and 'Mansoo' pear. Korean J. Hort. Sci. Technol. 22:55-62.

Husain, S.R., J. Gillard and P. Cullard. 1987. Hydroxyl radical scavenging activity of flavonoids. Phytochemistry 26:24892491.

Jang, U.B., H.J. Choi, H.S. Han, J.H. Park, J.H. Son, J.H. Bae, T.S. Seong, B.J. An, H.G. Kim and C. Choi. 2003. Chemical structure of polyphenol isolated from Korean pear (Pyrus pyrifolia Nakai). Korean J. Food Sci. Technol. 35:959-967.

Jeong, H.R., G.N. Choi, J.H. Kim, J.H. Kwak, Y.S. Kim, C.H. Jeong, D.O. Kim and H.J. Heo. 2010. Nutritional components and their antioxidative protection of neuronal cells of litchi (Litchi chinensis Sonn.) fruit pericarp. Korean J. Food Sci. Technol. 42:481-487.

Jin, Y.O. and W.S. Song. 2012. Antioxidant activity of Pyrus serotina fruit in different cultivars and parts. Korean J. Plant Res. 25:498-503 (in Korean).

Kang, Y.H. 2009. Phenolic compounds and antioxidant activity in cell wall materials from deodeok (Codonopsis lanceloata). Korean J. Food Sci. Technol. 41:345-349.

Kroon, P., and G. Williamson. 2005. Polyphenol: dietary components with established benefits to health? J. Sci. Food
Agric. 85:1239-1240.

Lee, J.H. 2000. Antioxidative activity and related compound of apple pomace. Korean J. Food Sci.Technol. 32:908-913.

Lim, D.K., U. Choi and D.H. Shin. 1996. Antioxidative activity of ethanol extract from Korean medicinal plants. Korean J. Food Sci. Technol. 28:83-89.

Moreno, M.I.N., M.I. Isla, A.R. Sampietro and M.A. Vattuone. 2000. Comparison of the free radical-scavenging activity of propolis from several regions of Argentina. J. Ethnopharmacology 71:109-114.

Myung, J.E. and I.K. Hwang. 2008. Functional components and antioxidative activities of soybean extracts. Korean Soybean Digest. 25:23-29.

Park, J.H., B.W. Kang, J.E. Kim, M.J. Seo, Y.C. Lee, J.H. Lee, W.H. Joo, Y.H. Choi, H.S. Lim, Y.K. Jeong and B.K. Lee. 2008. Effect of ethanol extract from peel of Citrus junos and Poncirus trifoliata on antioxidant and immune activity. J. Life Sci. 18:403-408.

Park, S.J., H.Y. Leeand D.H. Oh. 2003. Free radical scavenging effect of seed and skin extracts from campbell early grape (Vitis labruscana B.). J. Korean Soc. Food Sci. Nutr. 32:115-118.

Park, Y.G., S.H. Kim and H.G. Chung. 2007. Antioxidant potential in the fruits of Pyrus species (pear) in Korea. Korean J. Med. Crop Sci. 15:335-338.

Park, Y.S., B.W. Kim, T.C. Kim, H.G. Jang, S.U. Chon, J.Y. Cho, S.H. Jiang and B.G. Heo. 2008. Physiological activity of methanol extracts from Korean kiwi fruits. Korean J. Hort. Sci. Technol. 26:495-500.

Re, R., N. Pellegrini, A. Pannlala, M. Yang and C.A. RiceEvans. 1999. Antioxidant activity applying an improved ABTS radical cation decolorization assay. Free Radic. Biol. Med. 26:1231-1237.

Roh, B.K., J.Y. Kimand J.Y. Kim. 2005. Anti-oxidant activities of Punica granatum extracts. J. Soc. Cosmet. Scientists Korean 31:207-212.

Sharma, A. and S. Sehgal. 1992. Effect of domestic processing, cooking and germination on the trypsin inhibitor activity and tannin content of faba. Plant Food Human Nutrition 42:127231.

Tsao, R., R. Yang, J.C. Young and H. Zhu. 2003. Polyphenolic profiles in eight apple cultivars using high-performance liquid chromatography (HPLC). J. Agric. Food Chem. 51:6347-6353.

Valko, M., D. Leibfritz, J. Moncol, M.T.D. Cronin, M. Mazur 
and J. Telser. 2007. Free radicals and antioxidant in normal physiological functions and human disease. J. Biochem Cell Biology 39:44-84.

Wolfe, K., X. Wu and R.H. Liu. 2003. Antioxidant activity of apple peels. J. Agric. Food Chem. 51:609-614.

Yen, G.C. and H.Y. Chen. 1995. Antioxidant activity of various tea extracts in relation to their antimutagenicity. J. Agric.
Food Chem. 4:27-32.

Zhang, Y.B., M.J. Bae, B.J. Ahn, H.J. Choi, J.H. Bae, S. Kim and C. Choi. 2003. Effect of antioxidant activity and change in quality of chemical composition and polyphenol compound during long-term storage. Korean J. Food Sci. Technol. 35:115-120.

(Received 23 October 2012 ; Revised 26 December 2012 ; Accepted 25 January 2013) 